\title{
Atitudes alimentares de pacientes oncológicos hospitalizados
}

\author{
Eatting attitudes of hospitalized câncer patients
}

Actitudes alimentarias de los pacientes oncológicos hospitalizados

Luma Stella Teichmann Bazzan

ORCID: https://orcid.org/0000-0003-3245-515X

Universidade Federal de Santa Maria, Brasil E-mail: luma_bazzan@hotmail.com

Silvana Bastos Cogo

ORCID: https://orcid.org/0000-0002-1686-8459 Universidade Federal de Santa Maria, Brasil E-mail: silvana.cogo@ufsm.br

Graciele Pontes

ORCID: https://orcid.org/0000-0002-5298-449X

Universidade Federal de Santa Maria, Brasil E-mail: graci_pontes@hotmail.com

Graciela Dutra Sehnem

ORCID: https://orcid.org/0000-0003-4536-824X

Universidade Federal de Santa Maria, Brasil E-mail: graciela.sehnem@ufsm.br

Aline Ost dos Santos

ORCID: https://orcid.org/0000-0002-1849-9933

Universidade Federal de Santa Maria, Brasil E-mail: aline.ost@acad.ufsm.br

Jéssica da Rocha Mareque

ORCID: https://orcid.org/0000-0002-7809-8963

Universidade Federal de Santa Maria, Brasil

E-mail: jessicarmareque@ hotmail.com

Alice Marchezan

ORCID: https://orcid.org/0000-0002-5957-3650

Universidade Federal de Santa Maria, Brasil

E-mail: a.marchezan2@gmail.com

Natiely Lange da Silva

ORCID: https://orcid.org/0000-0003-4941-2586

Universidade Federal de Santa Maria, Brasil E-mail: natielylange.to@gmail.com

Taisa Nardi Fraga

ORCID: https://orcid.org/0000-0002-5822-2293

Universidade Federal de Santa Maria, Brasil E-mail: taisa_ta1@hotmail.com

\begin{abstract}
Resumo
Objetivo: Identificar as atitudes alimentares de pacientes oncológicos hospitalizados com doença progressiva e avançada. Metodologia: Trata-se de uma pesquisa qualitativa e exploratória, por meio de entrevistas semiestruturada e projetiva, baseada em questões norteadoras e com imagens, realizada em uma unidade de internação oncológica de um hospital universitário no sul do Brasil com pacientes em tratamento quimioterápico paliativo, no período de agosto de 2019 a janeiro de 2020. Resultados: Foram entrevistados cinco pacientes com faixa etária entre 45 e 79 anos. Para tanto, emergiram as categorias: dificuldades dietoterápicas após o diagnóstico do câncer; sentimentos relacionados a alimentação durante o processo de adoecimento e hospitalização; e a afetividade com a comida. Conclusão: Os pacientes oncológicos possuem atitudes alimentares positivas, levando em consideração o indivíduo na sua totalidade e no ambiente que lhe circula, pôde-se observar algumas estratégias de enfrentamento para o processo de adoecimento relacionado à alimentação. Percebe-se a relevância da temática e a necessidade de novas pesquisas e da equipe multidisciplinar de saúde habilitada para perceber e avaliar os sentimentos e as atitudes alimentares do paciente oncológico.
\end{abstract}

Palavras-chave: Cuidados Paliativos; Adaptação psicológica; Estado nutricional.

\section{Abstract}

Objective: To identify the food attitudes of hospitalized oncology patients with progressive and advanced disease. Methods: This is a qualitative and exploratory research, through semi-structured and projective interviews, based on 
guiding questions and with images, conducted in an oncology inpatient unit of a university hospital in southern Brazil with patients undergoing palliative chemotherapy treatment, in the period from August 2019 to January 2020. Results: Five patients with age range between 45 and 79 years were interviewed. For this, the categories emerged: dietary difficulties after cancer diagnosis; feelings related to food during the process of illness and hospitalization; and affectivity with food. Conclusion: Cancer patients have positive food attitudes, taking into account the individual as a whole and the environment that surrounds them, it could be observed some coping strategies for the process of illness related to food. We realize the relevance of the theme and the need for new research and for a interdisciplinary health team qualified to perceive and evaluate the feelings and eating attitudes of cancer patients.

Keywords: Palliative Care; Adaptation psychologique; Nutritional Status.

\section{Resumen}

Objetivo: Identificar las actitudes alimentarias de pacientes oncológicos hospitalizados con enfermedad progresiva y avanzada. Metodología: Se trata de una investigación cualitativa y exploratoria, a través de entrevistas semiestructuradas y proyectivas, basadas en preguntas orientadoras y con imágenes, realizadas en una unidad de hospitalización de oncología de un hospital universitario del sur de Brasil con pacientes en tratamiento de quimioterapia paliativa, en el período de agosto de 2019 a enero de 2020. Resultados: Se entrevistó a cinco pacientes con un rango de edad entre 45 y 79 años. Por lo tanto, surgieron las categorías: dificultades dietéticas tras el diagnóstico del cáncer; sentimientos relacionados con la alimentación durante el proceso de adopción y hospitalización; y la afectividad con la comida. Conclusión: Los pacientes oncológicos poseen actitudes alimentarias positivas, teniendo en cuenta el individuo en su totalidad y el ambiente en el que circula, se observan algunas estrategias de enfrentamiento para el proceso de adoctrinamiento relacionado con la alimentación. Se percibe la relevancia del tema y la necesidad de nuevas investigaciones y del equipo interdisciplinar de salud habilitado para percibir y evaluar los sentimientos y las actitudes alimentarias del paciente oncológico.

Palabras clave: Cuidados Paliativos; Adaptación Psicológica; Estado Nutricional.

\section{Introdução}

Os cuidados paliativos tem como objetivo melhorar a qualidade de vida tanto dos pacientes quanto dos familiares e cuidadores. Sob esta perspectiva os cuidados paliativos se constituem na prestação de atenção holística a indivíduos de todas as idades com graves sofrimentos relacionados à saúde, devido a doenças graves e, especialmente, daqueles que estão perto do fim da vida (Radbruch, et al, 2020). Neste sentido, por se tratar de uma doença agravante, o câncer pode apresentar dificuldades e desafios de adaptação em relação à doença, aos tratamentos e às vivências, por isso, as decisões acerca da melhor estratégia nutricional devem ser pautadas no respeito as decisões do paciente e sua família (Paz \& Martins, 2020). Estas impactam no uso de estratégias de enfrentamento a longo prazo durante a vivência de um profundo estressor, que é o câncer (Kim, et al, 2021). Percebe-se a relevância de identificar as atitudes alimentares é fundamental no enfrentamento do curso da doença.

A relação do homem com o alimento inicia no nascimento e não se extingue com a terminalidade de vida, necessitando respeitar as necessidades biológicas e as suas relações, bem como as socioantropológicas sem ignorar as condições clínicas e aspectos éticos desse processo (Duarte, Sousa, Feijó-Figueiredo \& Freire, 2020). Para tanto, a comida conecta as pessoas fisicamente, através do ato de comer, sendo este influenciado por diversos fatores determinante.

As experiências de vida envolvem a afetividade com os alimentos e na iminência de uma doença ameaçadora de vida não diminuem, incluindo recordações agradáveis que a comida desperta e o paciente oncológico experimenta são as sensações devido à ausência ou dificuldade de ingestão (Amorin, 2021). Tais pensamentos e sentimentos podem ser identificados por meio das atitudes alimentares, que consistem em crenças, pensamentos, sentimentos e comportamentos em relação à comida, e sofrem influências subjetivas, sociais, ambientais e culturais (Alvarenga, Scagliusi, \& Philippi, 2010) As atitudes alimentares englobam a relação com alimento e, portanto, o comer ou não comer, em resposta a sensações e emoções (Souza; Pisciolaro, Polacow, Cordás, \& Alvarenga, 2014). Além disso, para manter um bom estado nutricional, é fundamental estabelecer e manter atitudes alimentares positivas, confiantes, confortáveis e flexíveis (Satter, 2007) 
Diante dos apontamentos elencados, questionou-se quais seriam as estratégias de enfrentamento utilizadas pelos pacientes oncológicos hospitalizados com doença progressiva, avançada e incurável, frente ao cuidado multiprofissional? Desta forma, entendendo que a alimentação humana vai além da função biológica e fisiológica, pois não se resume apenas ao ato de comer, sendo este influenciado por diversos fatores determinantes, é que o estudo objetivou identificar as atitudes alimentares de pacientes oncológicos hospitalizados com doença progressiva e avançada.

\section{Metodologia}

Estudo exploratório de abordagem qualitativa realizado em hospital universitário que tem Equipe Matricial de Cuidados Paliativos localizado na região central do Rio Grande do Sul desenvolvido na unidade que atende pacientes com doenças hematológicas e oncológicas. Os participantes da pesquisa foram os pacientes internados para tratamento oncológico na Clínica Médica no período de agosto de 2019 a janeiro de 2020.

A amostra do estudo foi realizada de forma intencional, adotando-se os seguintes critérios de inclusão: maiores de 18 anos, com cognitivo preservado, capacidade de comunicação verbal e cientes do diagnóstico e prognóstico de doença oncológica progressiva, avançada e incurável. Foram excluídos os pacientes com doença hematológica devido a maior complexidade e diversidade do tratamento quimioterápico quando comparados com os cânceres sólidos e pacientes com alimentação exclusiva por via alternativa.

Os participantes da pesquisa foram indicados pela enfermeira responsável da unidade, quanto à irreversibilidade de cura da doença e conhecedores do seu diagnóstico e prognóstico. Dentre estes dez indicados para a pesquisa, cinco aceitaram participar. Após foi realizado a consulta e análise dos prontuários físicos e eletrônicos dos participantes da pesquisa. Foram coletadas as seguintes informações: diagnóstico, tratamento atual, doença pré-existente, medicamentos utilizados, peso, altura e via de alimentação. Assim, mediante a análise destas informações, do estado geral, dos critérios de inclusão e exclusão elencou-se cinco participantes em tratamento oncológico.

Para a entrevista foi proposto aos participantes da pesquisa se deslocarem para uma sala reservada, a fim de garantir o sigilo e a privacidade dos mesmos, porém somente dois participantes aceitaram deslocar-se até a sala. Outros dois participantes optaram em permanecer no leito do hospital juntamente com seus acompanhantes, afirmando não se sentirem incomodados com a presença de terceiros e um participante permaneceu no leito, pois estava um quarto individual e seu acompanhante retirou-se no momento da entrevista.

A entrevista semiestruturada foi norteada por questões que versavam sobre a hospitalização e atividades significativas, às atitudes alimentares, o impacto no cotidiano dos sintomas relacionados com a doença/tratamento e as intervenções odontológicas. $\mathrm{Na}$ entrevista projetiva foi disponibilizada dez imagens aos pacientes, sem nenhuma descrição ou sentido previamente atribuído, sendo os significados refletidos e referenciados pelos pacientes por meio da experiência pessoal e momento atual vivenciado. As entrevistas, duraram em média 45 minutos, foram gravadas em um gravador digital e posteriormente transcritas na íntegra. A identificação das atitudes alimentares nos pacientes oncológicos hospitalizados com doença progressiva e avançada ocorreu a luz da análise textual discursiva com quatro focos centrais: a desmontagem dos textos, o estabelecimento de relações, a captação do novo emergente e a construção de processo auto organizado (Moraes \& Galiazzi, 2020).

O estudo considerou os preceitos éticos do sigilo, confidencialidade e anonimato bem como suas ações receberam parecer favorável do Comitê de Ética em Pesquisa de Seres Humanos da Universidade Federal de Santa Maria, sob número 3.387.139. Este é oriundo do projeto intitulado "O enfrentamento dos pacientes oncológicos com doença ameaçadora de vida frente ao cuidado multiprofissional" sob o registro CAAE 13845219.400005346. 


\section{Resultados}

Foram entrevistados cinco pacientes com faixa etária entre 45 e 79 anos, destes três do sexo feminino e dois do sexo masculino. Todos os participantes estavam internados para realizar o tratamento quimioterápico paliativo. Além disso, três pacientes apresentaram perda de peso grave no período de 1 mês.

Para tanto, emergiram da análise das entrevistas três categorias: as adaptações dietoterápicas após o diagnóstico do câncer; os sentimentos relacionados a alimentação durante o processo de adoecimento e hospitalização; e a afetividade com a comida.

\section{Dificuldades dietoterápicas após o diagnóstico de câncer}

Os participantes referiram que necessitaram realizar alterações na alimentação após o diagnóstico e início do tratamento, em virtude dos sintomas adversos da quimioterapia, ou devido a cirurgia. Os principais sintomas mencionados que impactaram os hábitos alimentares foram: alteração no paladar (disgeusia), diminuição do paladar (hipogeusia), dificuldade ao deglutir (disfagia), sensação de salivação espessa e boca seca (xerostomia), constipação e inapetência (perda do apetite).

“Carne, eu tenho uma dificuldade muito grande de comer, principalmente de gado, galinha então nem se fala, não consigo (...) talvez o guisado assim eu tenho mais flexibilidade para comer (...) mudou bastante, eu tenho evitado de comer queijo, até por causa do intestino, para não prender" (P1).

“A alimentação para mim agora está sendo bem dificil por causa do medicamento que tira muito o paladar. Eu estou comendo o mínimo, eu não sinto vontade, já emagreci $13 \mathrm{~kg}$ depois que eu comecei. Me dá aquelas ânsias e eu não sinto vontade" (P2).

"Ultimamente eu tenho sentido um gosto muito esquisito na boca. (...) Deus me livre, parece que tem uma coisa queimando assim sabe, e antes da boca. (...) É, de repente é isso aí que as vezes eu não como tanta comida, eu não consigo." (P3).

"Nos dois últimos ciclos eu fiquei com mucosite, a cada ciclo, eu fiquei 12 dias sem comer, a base de líquidos. Até a água doía quando tomava” (P4).

“Olha, eu até que forçava um pouquinho mas não conseguia comer a mesma quantidade que eu comia antes. Então aí eu procurava um líquido que doía menos, um iogurte, um energético” (P5).

O impacto no cotidiano e escolhas alimentares foi significativo, sendo necessário adaptações percebidas como positivas e mais saudável.

“Eles (cozinheiros do hospital) usam produto para tirar as bactérias da salada, não tem jeito de conseguir comer. Eu já experimentei, e não tá fácil, nem em casa. Daí eu vejo que não quero e falo assim para o meu marido "vou deixar isso aqui, que não vou conseguir”. Aí eu vou estragar a vontade de comer as outras comidas” (P3).

"Mais comida integral, comidas líquidas também que eu não comia, comidas mais saudáveis, porque eu comia bastante comidas com condimentos, que não era o ideal"(P4). 
Sentimentos relacionados com a alimentação durante o processo de adoecimento e hospitalização

Surgiram diferentes significados/sentimentos dos pacientes em tratamento oncológico relacionados as adaptações na alimentação. Para tanto, sentimentos positivos foram associados a tranquilidade e aceitação das mudanças, ainda que o período de hospitalização interferisse na ingesta alimentar.

“Bem tranquila. Bem saudável (...) Só não fazendo quimio. Porque quando eu estou fazendo quimio, a comida do hospital não vai de jeito nenhum (...) Eu vou te dizer assim que depois da doença houve uma transformação muito grande. (...) Então eu tenho que entender não deu, outra hora vai dar”. (P4).

“Tudo é questão de te acostumar, no começo parece que tudo é dificuldade (...). A questão é essa, se tu não começar tu não vai terminar, tem que pelo menos começar, tomar coragem e começar”. (P5)

Sob outra perspectiva, sentimentos negativos como aborrecimento e frustração foram associados a desconfortos diante da redução da ingesta alimentar, indicando que o processo de hospitalização interfere nessa adaptação.

"Não me sinto bem porque tem coisas que eu não consigo comer, na hora do almoço. Em casa até eu tento comer, faço um bife bem feitinho, um arrozinho, feijãozinho, estou dizendo no diminutivo, porque é tudo pouquinho. Mas daí aos poucos eu vou voltando a minha alimentação. A minha dificuldade mesmo é aqui (hospital)” (P3).

"Eu me sinto meio chateado porque antes eu comia de tudo, não rejeitava nada. E agora eu me sinto meio reprimido nessa questão, eu acho que é por isso de repente, parece que o organismo perde naquele tipo de alimentação e eu não consigo comer, a gente fica frustrado"(P1).

Percebe-se o significado da alimentação como penoso, difícil, problemático devido aos efeitos da quimioterapia (náuseas, hipogeusia e inapetência), mas também obrigatório e relevante. Houve menção sobre a importância da alimentação correlacionada com sua composição, destacando os macros e micronutrientes (carboidratos, vitaminas e ferro), porém há verbalização que, especialmente durante a hospitalização, a ingesta alimentar torna-se algo penoso.

Tu sabes que eu comia de tudo, e bastante, e depois da cirurgia do esôfago diminuiu bastante, não consigo comer digamos, se eu comia um prato cheio, agora eu como meio prato e chega um momento que eu acho que o próprio organismo, detecta que eu já estou comendo o limite, começa a trancar o alimento (...) se eu força depois estou vomitando, fico bem ruim (P1).

(É importante) por causa das vitaminas, dos carboidratos e coisa que tem, que a gente precisa comer, de ferro (...) no feijão tem ferro, é importante, e o outro (arroz) tem também. E a gente sabe que uma comida seria bem importante, só que eu não tô conseguindo (P3).

Afetividade com a comida 
A afetividade com a comida foi associada a afeto, desejo, ressignificação, boas lembranças e a socialização. Embora algumas narrativas apresentam sentimentos negativos em relação à alimentação durante o período de adoecimento e hospitalização, também houve falas afetivas relacionadas à comida favorita.

Minha comida preferida hoje?! É o que (risos) eu não posso comer! Eu tô sonhando com um hambúrguer (...) Porque eu nunca mais comi (...) Eu comia antes. Eu nunca mais comi, então eu sinto vontade. Eu como bem o arroz, o feijão, o risoto, para mim hoje, viraram um hambúrguer $(P 4)$.

Um churrasco bem assadinho e uma maionese (...) porque eu sempre fui da carne assada (...) [Lembrança] A reunião de família, aniversário. Não tem como não ter lembrança. Para mim é bom as pessoas estarem juntos (P3).

Eu quando estava sadia eu comia tudo, de tudo (risos) não tenho preferência de comida. Só que agora, eu estou restrita, gostando mais de comer comida com caldinho, parece que está indo muito melhor do que as outras (P2).

\section{Discussão}

A nutrição em cuidados paliativos deve envolver os fatores culturais, emocionais além do fornecimento de alimentos e calorias (Lima \& De Lima, 2021). Para tanto, paliação é toda medida terapêutica que resulte em alívio permanente ou temporário de sofrimento (Palhares, Santos \& Cunha, 2018). A alimentação é essencial no auxílio nos âmbitos físico e mental ao paciente oncológico (Menezes, Carvalho, Borges \& Colares, 2019). A biografia do paciente oncológico, suas escolhas deve ser levada em consideração (Pereira \& Fernandes, 2020). Sob esta perspectiva, as atitudes alimentares bem como dificuldades e potencialidades devem ser levadas em consideração.

O paciente com câncer pode apresentar dificuldades e desafios de adaptação em relação à doença, aos tratamentos e às vivências. Desde o momento do diagnóstico, a doença é estigmatizada como sinônimo de sofrimento, perdas e preocupações, ou seja, cria-se uma situação e ambiente estressante para o paciente e sua rede de apoio (familiares e amigos), uma vez que os mesmos percebem uma quebra na rotina, o qual invariavelmente leva às mudanças de comportamento (Pereira \& Rodrigues, 2016).

Os indivíduos com câncer em progressão e estágio avançado, experimentam uma carga diversa e complexa de sintomas que são considerados de impacto nutricional, visto que comprometem a ingestão de alimentos, o peso e piora a qualidade de vida. Para tanto, é fundamental que o profissional conheça a melhor abordagem terapêutica e possibilite o bemestar, evitando intervenções desnecessárias que possam causar mais sofrimento ao paciente (Silva, et al, 2020). Sob este prisma, doenças ameaçadoras da vida implicam em acompanhamento ativo desde o diagnóstico (Lima, Maia \& Nascimento, 2019).

Percebe-se que a experiência destes sintomas em pacientes oncológicos tem grande impacto nutricional, pois reduzem o prazer pela comida e tornam a alimentação desagradável. Em estudo com pacientes com câncer em cuidados paliativos demonstrou a gravidade de 16 sintomas, com os três principais relacionados ao bem-estar reduzido, falta de apetite e fadiga. A gravidade de oito sintomas (falta de apetite, saciedade precoce, fadiga, diarreia, sabor anormal, dificuldade em engolir, sonolência, bem-estar reduzido), foram significativamente maiores no grupo de caquexia do que no grupo sem caquexia (Amano, et al, 2018).

Estes sintomas impactam na sensação de fome e na mudança de hábitos alimentares, visto que além da fome fisiológica, há a fome hedônica (apetite), que corresponde ao desejo de comer certos alimentos, e esta é muito sensível ao estresse e à qualidade gustativa dos alimentos, o que é normalmente afetado pelos tratamentos oncológicos. Embora todos os 
participantes do presente estudo necessitaram de alterações dietoterápicas, os mesmos possuem algumas estratégias de enfrentamento para as mudanças necessárias, podendo-se identificar a aceitação quanto a necessidade de mudanças, em que não estão estimulando sentimentos e comportamentos negativos para a alimentação, como a resistência, e visivelmente, isto deriva de atitudes alimentares positivas.

No viver está intrínseco a relação com os alimentos, incluindo as recordações agradáveis que certas comidas despertam. A relevância dessa relação não diminui na terminalidade de vida, entretanto se intensifica outras sensações causadas pela ausência ou dificuldade de ingestão (Amorim \& Silva, 2021)., como por exemplo, que sua sobrevivência está ameaçada, devido à piora da qualidade de vida. Acerca dos sentimentos negativos relacionados com a hospitalização, pode-se correlacionar com um estudo realizado com 423 pacientes internados na especialidade da oncologia, em que os participantes relataram que seu apetite é melhor em casa do que no hospital e a hospitalização impacta na perda de apetite (Ali, Dılşa, Filiz \& Güngör, 2017).

Sob esta perspectiva o alimento envolve aspecto biológico, social e cultural, repleto de significados (Duarte, et al, 2020). Os significados sobre o comer hoje na perspectiva dos participantes foram de dificuldade e obrigação. Entretanto, na percepção das narrativas, quando relembrados sobre sua comida mais prazerosa, há significado emocional e valor social, sendo importante no relacionamento familiar e socializações.

Além deste significado emocional está a obrigação de se alimentar, um estudo evidenciou que os pacientes com câncer frequentemente se tornam resolutos em seu desejo de manter uma alimentação adequada, forçando-se a comer apesar da dor, náusea e outros sintomas, da mesma maneira que relatado por participantes da atual pesquisa, dando um significado ao comer como imposição/obrigação (Amano, Baracos, \& Hopkinson, 2019). Percebe-se também alimentação como o significado de sobrevivência (Costa \& Soares, 2016).

Neste estudo, em relação a afetividade com a comida, os participantes relembraram sobre a sua comida favorita justificando-a como lembranças afetivas, desejos e ressignificações. Embora estes não consigam alimentar-se da mesma maneira, eles transformaram o que conseguem comer em algo agradável, tornando-se a comida favorita do momento, sendo está uma importante estratégia de enfrentamento através de uma relação positiva entre a alimentação e afetividade, trazendo significados prazerosos, lembranças e ressignificações.

\section{Conclusão}

Os pacientes oncológicos com doença em estágio avançado, progressivo e sem perspectiva de cura possuem atitudes alimentares positivas, uma vez que, deve-se levar consideração o indivíduo na sua totalidade e no ambiente que lhe circula, ou seja, os pacientes ainda conseguem apresentar algumas estratégias relacionadas à alimentação no processo de enfrentamento da doença, como por exemplo a aceitação às mudanças de comportamento, embora estejam passando por eventos estressores, de perda, necessidade de mudanças de comportamento e readaptações.

Percebe-se a relevância da temática bem como a necessidade de novas pesquisas e da equipe interdisciplinar de saúde habilitada para perceber e avaliar o quão os sentimentos negativos, estresse emocional e comportamentos de resistência relacionados com a alimentação podem estar interferindo na vida cotidiana e nas relações interpessoais do paciente, de maneira a aliviar o sofrimento do mesmo, ou dos familiares, sendo este um dos princípios dos cuidados paliativos.

Como limitações do estudo, salienta-se que, embora seja um estudo de caráter qualitativo, foi realizado com um número pequeno de amostra, pois houve algumas barreiras como o pequeno período de internação, procedimentos necessários realizados a qualquer o momento, como infusão de quimioterapia, e para muitos pacientes, a ausência do entendimento de seu prognóstico real, portanto, não pode-se generalizar as percepções e sentimentos deste grupo para todos os indivíduos que 
possuem diagnóstico de câncer em estágio avançado, progressivo e sem perspectiva de cura. Deve-se levar em consideração também, que há poucos autores que estudam sobre o tema abordado neste trabalho, e ainda assim, a grande minoria analisa sob a ótica do paciente.

\section{Referências}

Alvarenga, M., Scagliusi, F. B., \& Philippi, S. T. (2010). Development and validity of the Disordered Eating Attitude Scale (DEAS).Perceptual and motor skills,110(2), 379-395. https://doi.org/10.2466/PMS.110.2.379-395

Ali Alkan, Dılşa Mızrak, Filiz Çay Şenler, \& Güngör Utkan. (2017). Inadequate Nutritional Status of Hospitalized Cancer Patients.Journal of Clinical and Experimental Investigations,8(1), 15-21. https://doi.org/10.5799/ahinjs.01.2017.01.0616

Amano, K., Baracos, V. E., \& Hopkinson, J. B.. (2019). Integration of palliative, supportive, and nutritional care to alleviate eating-related distress among advanced cancer patients with cachexia and their family members.Critical Reviews in Oncology/hematology,143, 117-123. https://doi.org/10.1016/j.critrevonc.2019.08.006

Amano, K., Morita, T., Koshimoto, S., Uno, T., Katayama, H., \& Tatara, R. (2018). Eating-related distress in advanced cancer patients with cachexia and family members: a survey in palliative and supportive care settings. Supportive care in cancer : official journal of the Multinational Association of Supportive Care in Cancer,27(8), 2869-2876. https://doi.org/10.1007/s00520-018-4590-6

Amorim, G. K. D., \& Silva, G. S. N. D.. (2021). Nutricionistas e cuidados paliativos no fim de vida: revisão integrativa.Revista Bioética,29(3), 547-557. https://doi.org/10.1590/1983-80422021293490

Costa, M. F., \& Soares C., J. (2016). Alimentar e Nutrir: Sentidos e Significados em Cuidados Paliativos Oncológicos. Revista Brasileira De Cancerologia,62(3), 215-224. https://doi.org/10.32635/2176-9745.RBC.2016v62n3.163

Duarte, E. P. S., Sousa, R. R. de, Feijó-Figueiredo, M. C., Freire, J. A. P. (2020). Assistência Nutricional para os cuidados paliativos de pacientes oncológicos: uma revisão integrativa. Revista de Atenção à Saúde, 18, (64).124- 132 https://doi.org/10.13037/ras.vol18n64.6585

Kim, J. H. J., Bright, E. E., Williamson, T. J., Krull, J. L., Weihs, K. L., \& Stanton, A. L.. (2021). Transitions in coping profiles after breast cancer diagnosis: implications for depressive and physical symptoms.Journal of Behavioral Medicine,44(1), 1-17. https://doi.org/10.1007/s10865-020-00159-w

Lima Mascarenhas, H., \& De Lima Costa, A. R.. (2021). Até quando o nutrir o paciente oncológico em cuidados paliativos. Uma revisão integrativa. Health Residencies Journal - HRJ,2(10), 76-90. https://doi.org/10.51723/hrj.v2i10.97

Lima, K. M. D. A., Maia, A. H. N., \& Nascimento, I. R. C. D.. (2019). Comunicação de más notícias em cuidados paliativos na oncopediatria. Revista Bioética, 27(4), 719-727. https://doi.org/10.1590/1983-80422019274355

Menezes, S. A. T. D., Carvalho, A. C. L. C. D., Borges, K. M., Colares, R. A., Albuquerque, V. P. G. E., \& Bueno, M. M.. (2019). Dietoterapia e cuidados paliativos em pacientes oncológicos. ID on Line. Revista De Psicologia,(46), 40-41. https://doi.org/10.14295/idonline.v13i46.2007

Moraes R., Galiazzi M. C.(2020). Análise Textual Discursiva. Unijuí.

Palhares, D., Santos, Í. A. D., \& Cunha, A. C. R. D. (2018). Suspensão de nutrição enteral a pacientes em coma persistente. Revista Bioética,26(2), 251-259. https://doi.org/10.1590/1983-80422018262246

Paz, Á. S., Silva, B. F. G. D., \& Martins, S. S.. (2020). Nutrição em cuidados paliativos oncológicos: Aspectos bioéticos. Brazilian Journal of Health Review,3(4), 8891-8903. https://doi.org/10.34119/bjhrv3n4-134

Radbruch, L., De Lima, L., Knaul, F., Wenk, R., Ali, Z., Bhatnaghar, S., Blanchard, C., Bruera, E., Buitrago, R., Burla, C., Callaway, M., Munyoro, E. C., Centeno, C., Cleary, J., Connor, S., Davaasuren, O., Downing, J., Foley, K., Goh, C., Gomez-Garcia, W., Pastrana, T. (2020). Redefining Palliative Care-A New Consensus-Based Definition.Journal of pain and symptom management, 60(4), 754-764. https://doi.org/10.1016/j.jpainsymman.2020.04

Satter, E. (2007.). Eating Competence: Definition and Evidence for the Satter Eating Competence Model.Journal of Nutrition Education and Behavior,39, S142-S153.

Pereira, C. D. A., \& Fernandes De Souza Ribeiro, J.. (2020). Cuidados paliativos: Reflexões sobre a Psicologia e os cuidados paliativos para pacientes e familiares.Revista Mosaico,10(2Sup), 111-115. https://doi.org/10.21727/rm.v10i2sup.1826

Pereira, T. B., \& Rodrigues Branco, V. L.. (2016). As Estratégias de Coping na Promoção à Saúde Mental de Pacientes Oncológicos: Uma Revisão Bibliográfica.Revista Psicologia E Saúde,1(8). https://doi.org/10.20435/2177093x2016104

Silva, M. P. B., Leite, A. C., Cunha, F. C. G., Soares, A. R. G., Silva, L. D. L., Lima, J. H. C., Almeida, F. S. D., Loures, L. L. D. S., Pereira, K. V. D. S. A., Fernandes, C. R. S., Costa, G. O. P. D., Macêdo, L. S., Oliveira, M. D. S., Lima, M. S., Sousa, K. C. D., Câmara, J. T., Rodrigues, D. P., Pinto, D. C. C., Ribeiro, A. M. N., ... Oliveira, D. N.. (2020). Terapia nutricional em pacientes oncológicos em cuidados paliativos.Research, Society and Development,9(10), e9309109326. https://doi.org/10.33448/rsd-v9i10.9326

Souza, A. C. D., Pisciolaro, F., Polacow, V. O., Cordás, T. A., \& Alvarenga, M. D. S.. (2014). Atitudes em relação ao corpo e à alimentação de pacientes com anorexia e bulimia nervosa.Jornal Brasileiro De Psiquiatria, 63(1), 1-7. https://doi.org/10.1590/0047-2085000000001 